\title{
Prostate Neuroendocrine Carcinoma
}

National Cancer Institute

\section{Source}

National Cancer Institute. Prostate Neuroendocrine Carcinoma. NCI Thesaurus. Code C158912.

A small cell or rarely a large cell neuroendocrine carcinoma that arises from the prostate gland. 